\title{
Die Goldlärche (Pseudolarix amabilis), ein attraktives Ziergehölz aus China
}

\author{
Veit Martin Dörken \& Annette Höggemeier
}

\begin{abstract}
The golden larch (Pseudolarix amabilis) is an ornamental eastern Asian conifer with a long evolutionary history. It is one of the few deciduous gymnosperms which sheds its leaves after an intensive golden autumnal coloring.
\end{abstract}

\section{Zusammenfassung}

Die Goldlärche (Pseudolarix amabilis) ist eine ostasiatische Konifere mit einer langen evolutionären Geschichte. Den volkstümlichen Namen kann man erst im Herbst verstehen, wenn sich die Nadeln vor dem Abwerfen in ihrer prächtig leuchtenden Herbstfärbung präsentieren.

\section{Einleitung}

In der mitteleuropäischen Gartenkultur ist die Goldlärche (Pseudolarix amabilis, Pinaceae) recht unbekannt. In Ostasien dagegen wird sie häufig kultiviert und dort besonders in großen Parkanlagen als wertvolles Solitärgehölz (vor allem wegen der goldenen Herbstfärbung) gepflanzt. Darüber hinaus ist sie auch gelegentlich in Tempelanlagen anzutreffen. Nicht nur als Ziergehölz für die Gartenkultur, sondern auch aus botanischer Sicht ist die Goldlärche in mehrfacher Hinsicht ein besonders interessantes Gehölz.

\section{Verbreitung}

Die Goldlärche, die von Robert Fortune, einem bedeutenden englischen Pflanzensammler, 1852 nach Europa eingeführte wurde, stammt aus kleinen disjunkten Arealen. Diese reichen in Ost- und Zentralchina von Ost-Setschuan weiter östlich bis Südwest-Jiangsu, Zhejiang und Tentral-Gujan. In diesem Areal wächst die Goldlärche in Höhenlagen von 100-1500 (-2300) m (ECKenwalder, 2009). Sie ist dort mit zahlreichen winterkahlen Laubbäumen und anderen Koniferen vergesellschaftet.

Die ältesten bekannten Fossilien von Pseudolarix sind aus dem späten Jura bzw. der frühen Kreidezeit aus Südost-Russland und Nordost-China nachgewiesen. Somit handelt es sich bei Pseudolarix (neben Pinus) um eine der ältesten Kieferngewächse, die heute noch existieren (Le Page \& Basinger 1995; Nan 1995, Mai 2007; JaHren 2007; ZanNi \& RaVAZZi 2007;
Gernandt et al. 2008, Teodoridis \& Sakala 2008, Кıм 2009). Im Tertiär hatte die Gattung Pseudolarix ein wesentlich größeres Verbreitungsareal, als es das heutige noch vermuten lässt. So war sie auch in der Arktis und in Teilen Europas heimisch.

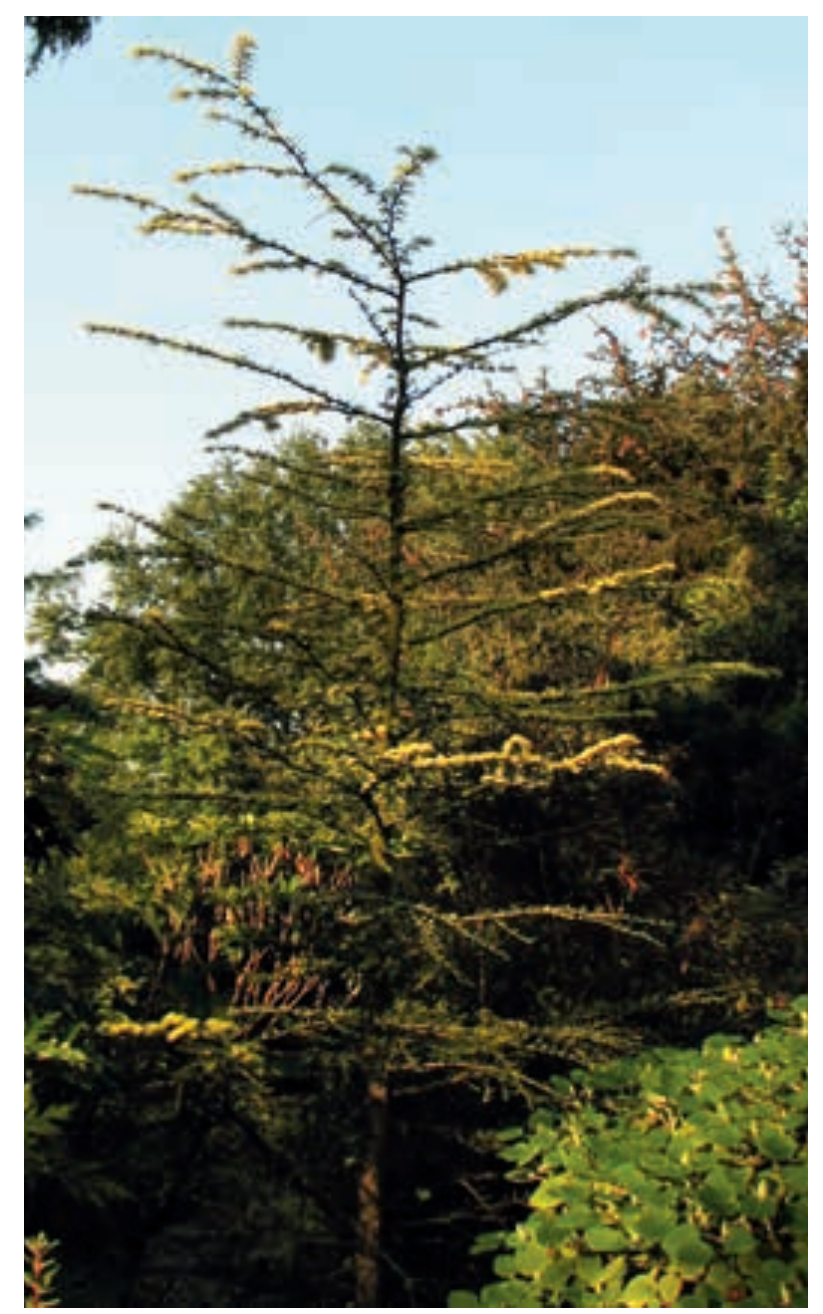

Abb. 1. Habitus der Goldlärche. 


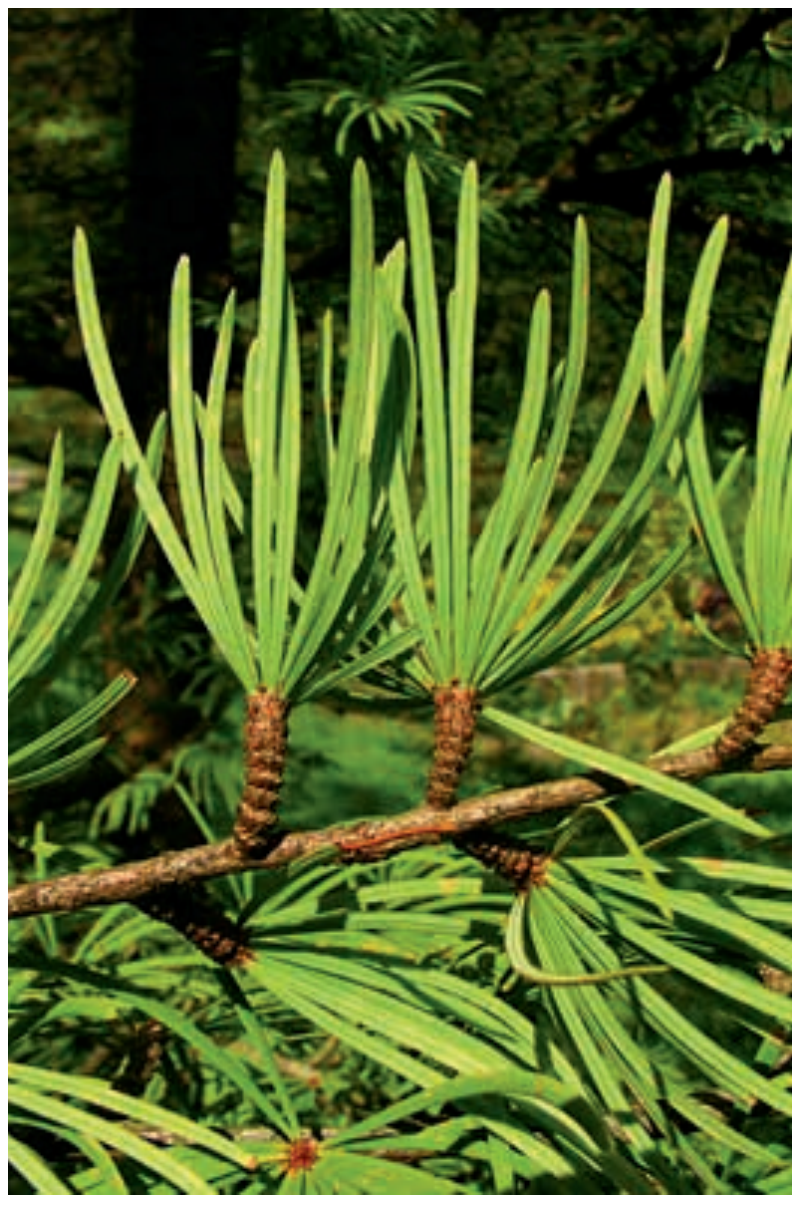

Abb. 2: Zweig mit Kurztrieben.

\section{Verwandtschaft}

Als Vertreter der Kieferngewächse ist die Goldlärche eng mit Tanne, Kiefer, Fichte, Lärche, Douglasie und Hemlocktanne verwandt. Der Gattungsname Pseudolarix stammt aus dem Griechischen und bedeutet übersetzt Scheinlärche, was darauf hindeutet, dass es sich nicht um eine „echte" Lärche der Gattung Larix handelt. Pseudolarix umfasst nur die einzige Art $P$. amabilis (FARJON 2001, 2010). Die Goldlärche wurde nach früheren Auffassungen zusammen mit den immergrünen Zedern (Cedrus) und den winterkahlen Lärchen (Larix) zur Unterfamilie der Laricoideae (den Lärchenartigen) zusammengefasst. Neuere morphologische und molekularphylogenetische Untersuchungen (z. B. WANG et al. 2000) belegen jedoch einen engen Bezug zu den Hemlocktannen (Tsuga) und Scheinhemlocktannen (Nothotsuga).

\section{Morphologie}

Goldlärchen werden 30 (-40) m hoch. Sie zeigen eine deutliche Differenzierung in Lang- und Kurz-

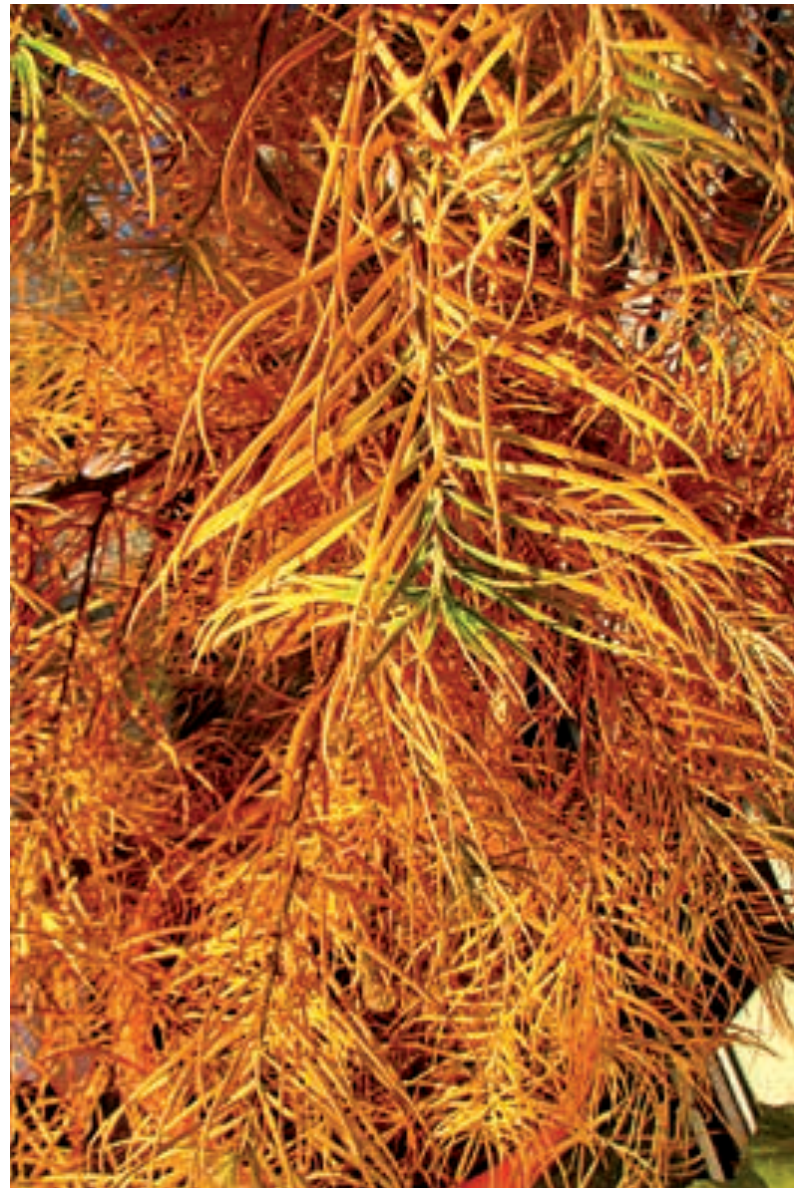

Abb. 3: Goldbraune Herbstfärbung.

triebe. Dabei stehen die Kurztriebe immer seitlich in einer Ebene an den Langtrieben, so dass mit zunehmendem Alter eine fischgrätenartige Verzweigung entsteht (Unterschied zu den echten Lärchen, hier stehen die Kurztriebe unregelmäßig am Langtrieb verteilt). Auch die ältesten bekannten Fossilien von Pseudolarix zeigen diesen sehr deutlich ausgeprägten Sprossdimorphismus (LE Page 1995). Basierend auf den fossilen Funden konnten bislang nur zwei unterschiedliche Pseudolarix-Arten identifiziert werden, eine davon ist der rezenten $P$. amabilis extrem ähnlich. Daher ist die Gattung Pseudolarix ein exzellentes Beispiel für eine morphologische Unveränderlichkeit über viele Millionen Jahre über verschiedene geologische Zeitalter hinweg. Es handelt sich um ein so genanntes lebendes Fossil. Ähnliches ist z. B. auch vom Urwelt-Mammutbaum (Metasequoia glyptostroboides) bekannt (ANDREws 1948). Dessen fossiler Vorläufer M. occidentalis ist so gut wie identisch mit der aktuell existierenden Art (Schweitzer 1980; Le Page et al. 2005). 


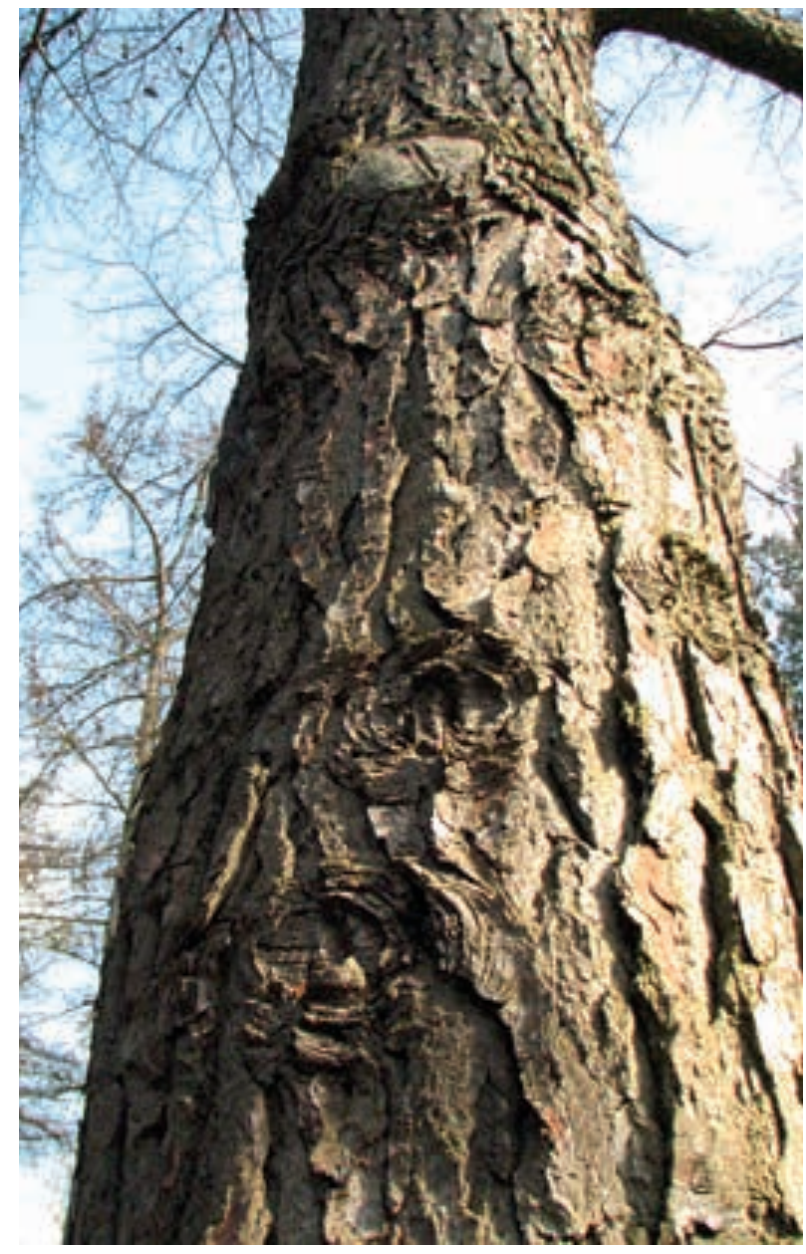

Abb. 4: Stamm mit rissiger Borke.
Die bis $8 \mathrm{~cm}$ langen und bis $0,3 \mathrm{~mm}$ breiten Nadeln sitzen den Langtrieben schraubig mit deutlich erkennbaren Internodien an. An den Kurztrieben entwickeln sie sich in dichten Spiralen. Die Nadeln der Goldlärche sind im Vergleich zu denen der echten Lärchen deutlich länger und breiter. Ihre Oberseite ist dunkelgrün, die Unterseite ist heller und weist zwei Spaltöffnungsbänder auf. Die Herbstfärbung ist ein leuchtendes Goldgelb.

Die Goldlärche ist wie auch alle anderen Kieferngewächse einhäusig. Die reproduktiven Strukturen sind in getrenntgeschlechtlichen Zapfen zusammengefasst. Die männlichen Pollenzapfen sind klein und länglich-elliptisch. Die verkehrt-eiförmigen, bis $6 \mathrm{~cm}$ langen weiblichen Samenzapfen stehen aufrecht und sind deutlich in Deck- und Samenschuppen gegliedert. Je Samenschuppe werden zwei Samenanlagen hervorgebracht. Im reifen Zustand ist die länglich-eiförmige, mehr oder weniger spitz zulaufende Samenschuppe meist doppelt so lang wie die Deckschuppe. Zur optimalen Ausbreitung der geflügelten Samen zerfallen die Zapfen und bleiben nicht (wie bei echten Lärchen) über Jahre am Baum erhalten. Ähnliches ist auch von Zedern und Tannen bekannt.

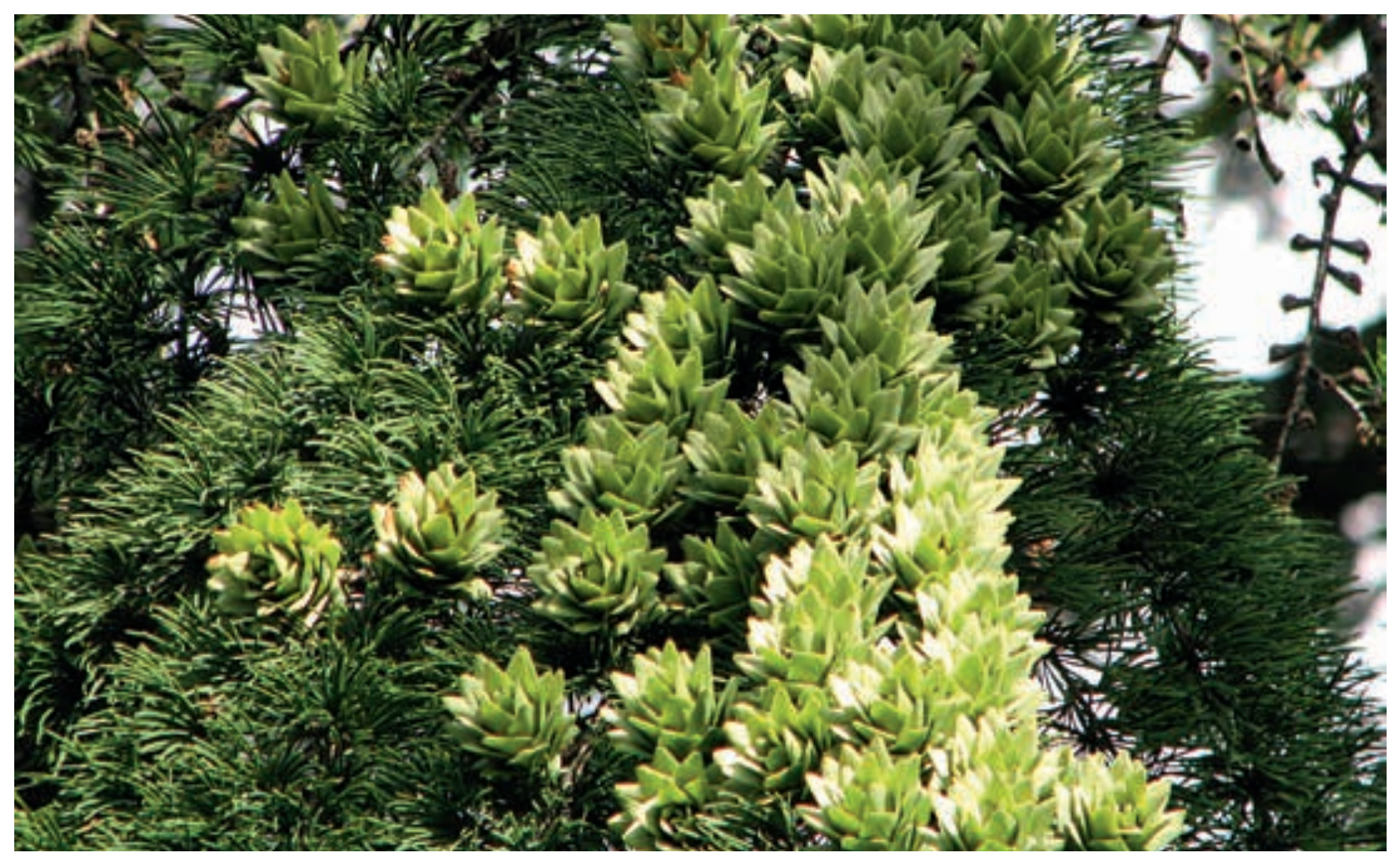

Abb. 5: Zweig mit jungen Zapfen. 


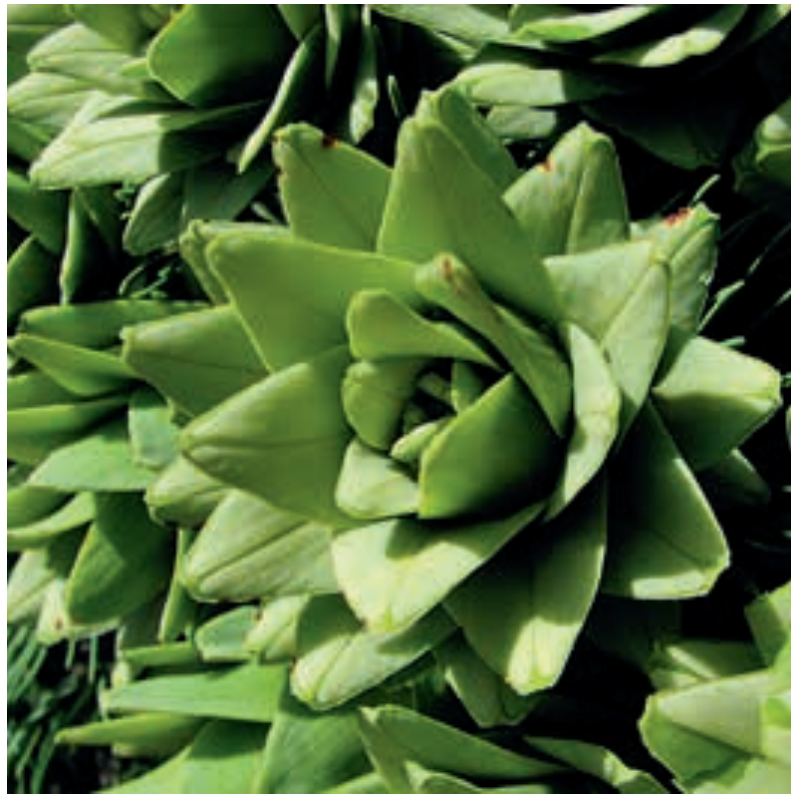

Abb. 6: Junger, noch grüner Zapfen der Goldlärche.

\section{Kulturansprüche}

Für ein optimales Gedeihen benötigt die Goldlärche frische bis feuchte Böden mit saurer Bodenreaktion. Empfindlich reagiert sie gegenüber Bodenverdichtungen und stark alkalischer Bodenreaktion. Auf schweren Lehm- und Tonböden vergreist die Goldlärche frühzeitig oder fällt ganz aus. Obwohl sie in Höhenlagen bis $1500 \mathrm{~m}$ vorkommt, ist sie besonders in ihrer Jugend frostempfindlich (Dörken \& Höggemeier 2009). Bei der Pflanzung sollte dies berücksichtigt werden, gegebenenfalls durch die Pflanzung von Schutzgehölzen in der Jugendphase.

\section{Literatur}

Andrews, H N. 1948: Metasequoia and the living fossils. Miss. Bot. Garden Bull. 36: 79-85.

Dörken, V.M. \& Höggemeier, A. 2009: Botanisch-dendrologische Streifzüge. Gehölzführer des Botanischen Gartens Bochum. - Bochum.

Eckenwalder, J. E. 2009: Conifers of the world. Portland.

FARJON, A. 2001: World checklist and bibliography of conifers, $2^{\text {nd }}$ ed. - Kew.

FARJON, A. 2010: A handbook of the world's conifers, Vol. II. - Brill, Leiden, Boston.

Gernandt, D.S., Magallón, S., López, G.G., Flores, O.Z., Willyard, A. \& Liston, A. 2008: Use of simultaneous analyses to guide fossil-based calibrations of Pinaceae phylogeny. - Int. J. Plant. Sci. 169: 1086-1099.

JAHREN, A.H. 2007: The arctic forest of the middle Eocene. Annu. Rev. Earth Planet. Sci. 35: 509-540.

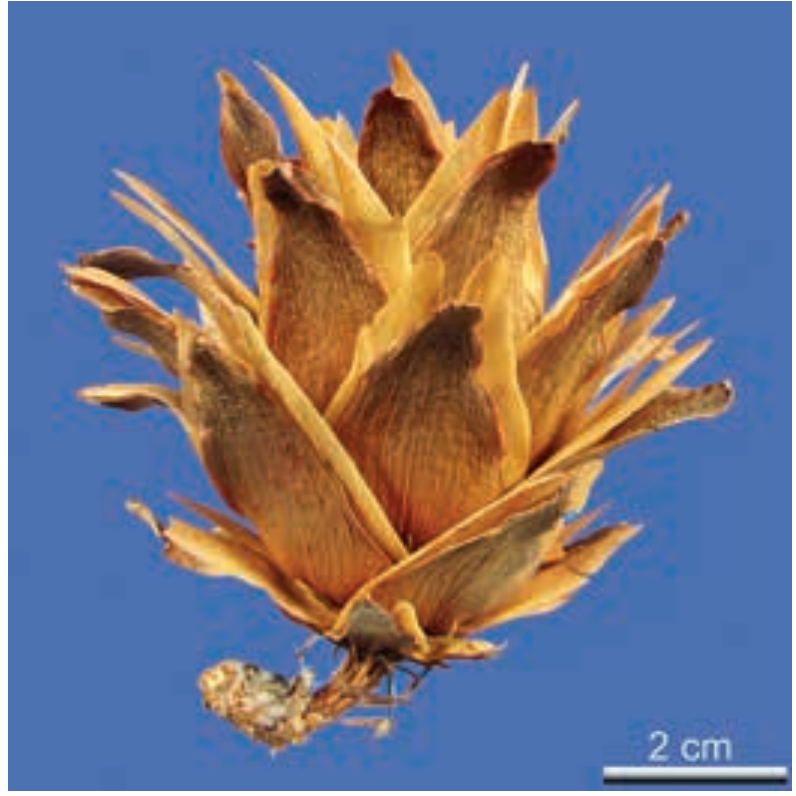

Abb. 7: Ausgereifter Zapfen.

Kim, J.-H. 2009: Occurrence of Pseudolarix (Pinaceae) from the Miocene Duho Formation of the Yeonil Group in the Pohang Basin, Korea. - J. Korean Earth Science Society 30: 598-604.

Le Page, B.A. \& Basinger, J.F. 1995: Evolutionary history of the genus Pseudolarix Gordon (Pinaceae). - Int. J. Plant Sci. 156: 910-950.

Le Page, B. A., Yang, H. \& Matsumoto, M. 2005: The evolution and biogeographic history of Metasequoia. - In: Le Page, B. A., Williams, C. J. \& Yang, H. (Hrsg.): The geobiology and ecology of Metasequoia. - Berlin, Heidelberg. MAI, D. H. 2007: The floral change in the Tertiary of the Rhön mountains (Germany). - Acta Palaeobotanica 47: 135-43.

NAN, L. 1995: Studies on the geographic distribution, origin and dispersal of the family Pinaceae Lindu. - Acta Phytotax. Sin. 33: 105-130.

Schweitzer, H.J. 1980: Environment and climate in the early Tertiary of Spitsbergen. - Palaeogeogr., Palaeoclimatol., Palaeoecol. 30: 297-311.

Teodoridis, V. \& Salaka, J. 2008: Early Miocene conifer macrofossils from the Most Basin (Czech Republic). - N. Jb. Geol. Paläont. Abh. 250: 287-312.

WANG, X-Q., TANK, D.C \& SANG, T. 2000: Phylogeny and divergence time in Pinaceae: evidence from three genomes. Molec. Biol. Evol. 17: 773-781.

Zanni, M. \& Ravazzi, C. 2007: Description and differentiation of Pseudolarix amabilis pollen palaeoecological implications and new identification key to fresh besaccate pollen. - Rev. Palaeobot. Palynol. 145: 35-75. 